\title{
Correction to: Diagnosis and treatment of Chiari Malformation and syringomyelia in adults: International Consensus Document
}

\author{
Palma Ciaramitaro ${ }^{1,2}$ - Luca Massimi $^{3} \cdot$ Alessandro Bertuccio $^{4} \cdot$ Alessandra Solari $^{5} \cdot$ Mariangela Farinotti $^{5}$. \\ Paola Peretta $^{6} \cdot$ Veronica Saletti $^{7} \cdot$ Luisa Chiapparini $^{8} \cdot$ Andrea Barbanera $^{4} \cdot$ Diego Garbossa $^{1} \cdot$ Paolo Bolognese ${ }^{9}$. \\ Andrew Brodbelt ${ }^{10} \cdot$ Carlo Celada $^{11}$. Dario Cocito ${ }^{12}$. Marcella Curone ${ }^{13} \cdot$ Grazia Devigili $^{14} \cdot$ Alessandra Erbetta $^{15}$. \\ Marilena Ferraris ${ }^{16}$. Marika Furlanetto ${ }^{17}$. Mado Gilanton ${ }^{18}$. George Jallo ${ }^{19} \cdot$ Marieta Karadjova $^{20}$. Jorg Klekamp ${ }^{21}$. \\ Fulvio Massaro ${ }^{22}$. Sylvia Morar ${ }^{23}$. Fabrice Parker ${ }^{23}$. Paolo Perrini ${ }^{24}$. Maria Antonia Poca ${ }^{25}$. Juan Sahuquillo ${ }^{25}$. \\ Marcus Stoodley ${ }^{26}$. Giuseppe Talamonti ${ }^{27}$ - Fabio Triulzi ${ }^{28}$. Maria Consuelo Valentini ${ }^{16}$ - Massimiliano Visocchi ${ }^{29}$. \\ Laura Valentini ${ }^{17}$. On behalf of the International Experts Jury of the Chiari Syringomyelia Consensus Conference, \\ Milan, November 11-13, 2019
}

Published online: 17 November 2021

(c) Fondazione Società Italiana di Neurologia 2021

Correction to: Neurological Sciences (2021)

https://doi.org/10.1007/s10072-021-05347-3

The original published online version does not include the full list of authors. The correct author names are given above.

The original article has been corrected.

Publisher's note Springer Nature remains neutral with regard to jurisdictional claims in published maps and institutional affiliations.

The original article can be found online at https://doi.org/10.1007/ s10072-021-05347-3

Palma Ciaramitaro

palma.ciaramitaro@gmail.com

1 CRESSC, SSD Coordinamento Neurofisiologia Clinica and Neurosurgery Unit, Department of Neuroscience, University of Torino, Torino, Italy

2 Neuroscience Dpt, Azienda Ospedaliero-Universitaria Città della Salute e della Scienza di Torino, Presidio CTO, via Zuretti, 29 Torino, Italy

3 Pediatric Neurosurgery, Fondazione Policlinico Universitario A. Gemelli IRCCS, Rome, Italy

4 Department of Neurosurgery, "SS Antonio e Biagio e Cesare Arrigo" Hospital, Alessandria, Italy
5 Neuroepidemiology Unit - Scientific Directorate, Fondazione IRCCS Istituto Neurologico Carlo Besta, Milan, Italy

6 Pediatric Neurosurgery, Ospedale Infantile ReginaMargherita, AOU Citta' della Salute e della Scienza di Torino, Torino, Italy

7 Developmental Neurology Unit, Fondazione IRCCS Istituto Neurologico Carlo Besta, Milan, Italy

8 Department of Neuroradiology, Fondazione IRCCS Istituto Neurologico Carlo Besta, Milan, Italy

9 Chiari Neuosurgical Center, Mount Sinai, South Nassau, Oceanside, NY, USA

10 Consultant Neurosurgeon, The Walton Centre NHS Foundation Trust, Liverpool, UK

11 AISMAC, Torino, Italy

12 Istituti Clinici Scientifici Maugeri, Torino, Italy 
13 Casa di Cura del Policlinico, Igea Headache Center, Milan, Italy

14 Department of Clinical Neuroscience, Fondazione IRCCS Istituto Neurologico Carlo Besta, Milan, Italy

15 Service of Neuroradiology, Fondazione IRCCS Istituto Neurologico Carlo Besta, Milan, Italy

16 Service of Neuroradiology, Diagnostic Imaging Department, AOU Citta' della Salute e della Scienza di Torino, Torino, Italy

17 Department of Neurosurgery, Fondazione IRCCS Istituto Neurologico Carlo Besta, Milan, Italy

18 APAISER, Nantes, France

19 Johns Hopkins University Department of Neurosurgery, Johns Hopkins Hospital, Baltimore, MD, USA

20 Neurology Department, University of Sofia, Sofia, Bulgaria

21 Christliches Krankenhaus Quakenbrück, Department of Neurosurgery, Quakenbrück, Germany
22 Department of Neurosurgery, University of Torino, Torino, Italy

23 Neurosurgery Department, Reference Center Rares Diseases C-MAVEM, CHU Bicetre APHP, Paris, France

24 Department of Translational Research and of New Surgical and Medical Technologies, University of Pisa, Pisa, Italy

25 Neurosurgery and Pediatric Neurosurgery, Vall d'Hebron Hospital Universitari, Neurotrauma and Neurosurgery Research Unit, and Universitat Autònoma de Barcelona, Barcelona, Spain

26 The Australian School of Advanced Medicine, Macquarie University, Macquarie Park, NSW 2109, Australia

27 Department of Neurosurgery, ASST Niguarda, Milan, Italy

28 Neuroradiology Unit, Fondazione IRCCS Ca Granda Ospedale Maggiore Policlinico, Milan, Italy

29 Department of Neurosurgery, Catholic University School of Medicine, Rome, Italy 